\title{
A Vaccine Kit for Prevention and Therapy of Alzheimer's Disease in a Transgenic Mouse Model
}

\author{
Iván Carrera $^{1 *}$, Carmen Vigo ${ }^{2}$ and Ramón Cacabelos ${ }^{1}$ \\ ${ }^{1}$ EuroEspes Biomedical Research Center, Institute for CNS Disorders and Genomic Medicine, Corunna 15166, Spain; ${ }^{2}$ Atlas Pharmaceu- \\ ticals, Sunnyvale 94089, CA, USA
}

\begin{abstract}
Background and objective: Alzheimer's disease (AD) is a major health problem in developed countries. The absence of specific biomarkers for diagnosis and the multiple limitations of available screening methods have slowed down the efficient implementation of AD population-based screening programs. Based on recent developments, we have designed and tested a new $A D$ vaccine tool kit as an effective framework for large-scale animal model screening. The $A D$ vaccine tool kit was developed for transgenic animal models of $A D$ in order to evaluate their neuropathological characteristics via multiple screening strategies.
\end{abstract}

Methods: This vaccine consists of the inoculation of a new immunogen-adjuvant designed to specifically activate antibodies against the generation of neuritic plaques produced by the toxic excess of amyloid- $\beta(A ß)$ and simultaneously to deal with the autoimmune activation that triggers acute meningoencephalitis, as observed in human trials in the past.

Results: In the present study, we show the current status of the AD vaccine tool kit, with a special focus on the evaluated data and its available settings. We also review the strengths and limitations of this kit in the context of its applicability in experimental research.

Conclusions: We focus our attention on the 'framework experimental immunization guidelines' for AD screening, which have recently been boosted by numerous research lines implemented throughout the scientific community.

Introduction

Alzheimer's disease (AD) is the most common type of dementia, affecting multiple brain functions. ${ }^{1}$ The exact cause of $\mathrm{AD}$ is still unknown, although some factors are thought to increase the risk of developing this progressive neurological disease. These factors include increasing age, a genetic-risk family history, previous severe

Keywords: Alzheimer's disease; Amyloid beta peptide; Transgenic mice; Immunotherapy; $\mathrm{AD}$ vaccine kit.

Abbreviations: $\mathrm{AD}$, Alzheimer's disease; $\mathrm{A} \beta, \beta$-amyloid; APP, amyloid precursor protein; APP/PS1, double transgenic model; GFAP, glial fibrillary acidic protein; IL, interleukin; PBS, phosphate-buffered saline; PDAPP, mouse model of Alzheimer's disease; S1P, sphingosine-1-phosphate; TBS, Trizma-buffered saline; TBS-T, TBS containing $0.1 \%$ Tween-20; Tg, transgenic; Th, T helper.

Received: January 02, 2018; Revised: January 16, 2018; Accepted: January 19, 2018

${ }^{*}$ Correspondence to: Iván Carrera, Department of Health Biotechnology, EuroEspes Biomedical Research Center, 15165 Bergondo, Corunna, Spain. Tel: +34 981780505; E-mail: biotecnologiasalud@ebiotec.com

How to cite this article: Carrera Iá, Vigo C, Cacabelos Ró. A Vaccine Kit for Prevention and Therapy of Alzheimer's Disease in a Transgenic Mouse Model. J Explor Res Pharmacol 2018;3(1):12-18. doi: 10.14218/JERP.2018.00004. head injuries, adverse lifestyle and cardiovascular aspects. ${ }^{2,3}$ The neuropathological hallmarks in the affected brain are characterized by the accumulation of extracellular amyloid- $\beta(\mathrm{A} \beta)$ peptide in amyloid plaques and intracellular neurofibrillary tangles with hyperphosphorylated tau proteins and massive neuronal-synaptic loss, affecting particularly the neocortex, hippocampus and entorhinal regions. ${ }^{4}$

In $\mathrm{AD}$ preclinical studies that have focused on basic research and drug discovery, animal models (mice in particular) are essential tools for uncovering biological mechanisms, validating molecular targets and screening potential immunization compounds. ${ }^{5-7}$ Both transgenic (Tg) and nongenetically-modified mouse models enable access to different types of AD-like pathology in vivo, crucial for gaining knowledge on disease etiology and to study the multiple AD interventions measured by targeting biomarkers. Over the last decade, the scientific community has been taking advantage of the potential aspects of double- and triple- $\mathrm{Tg}$ mouse lines, used to study emergent therapies for the prevention and reduction of the neurodegenerative features of $\mathrm{AD}$, in order to extrapolate later to human studies. ${ }^{8}$ Among these, active and passive immunization procedures against $\mathrm{A} \beta$ have been widely inves- 


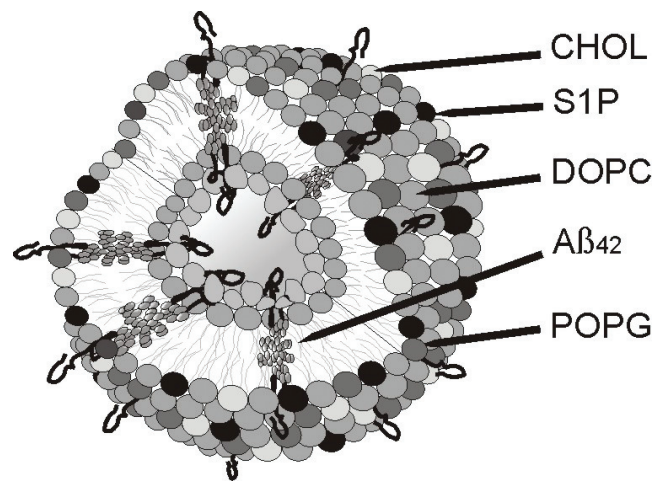

(EB101) : Aß42+S1P+L

Fig. 1. Biophysical illustration of the EB101 vaccine liposome. Structural representation of AB1-42/S1P-containing liposome (EB101). Image adapted from Carrera et al., 2015. ${ }^{15}$

tigated as potential immunotherapeutic approaches. ${ }^{9,10}$ However, until now, the $\mathrm{AD}$ vaccines tested in mice and humans have failed to control the progress of $\mathrm{AD}$ neuropathology, due to a massive $\mathrm{T}$ cell activation that results in a meningoencephalitis-like reaction. ${ }^{11}$

The purpose of this paper is to present a novel AD vaccine tool kit, that allows preclinical investigators and biomedical researchers to test its efficacy on different animal models of AD. Moreover, the $\mathrm{AD}$ vaccine tool kit was also designed to be a useful tool in the developmental process of improving our understanding of the pathology of $\mathrm{AD}$ in Tg models. This experimental tool is formed by the components of the immunogenic vaccine EB101, with a positive effect on the immunotherapeutic response of AD-like pathology in mouse models. ${ }^{12-17}$ Therefore, this effective vaccine was formulated to reduce $A \beta$ burden by generating robust anti$\mathrm{A} \beta$ antibody production, preventing or slowing down the AD-like pathological alterations and stimulating an anti-inflammatory $\mathrm{T}$ helper (Th) 2 immune response. ${ }^{18-20}$

\section{Materials and methods}

This innovative $\mathrm{AD}$ vaccine tool kit consists of several doses of immunotherapeutic inoculation compound, designed to specifically activate antibodies against the generation of neuritic plaques produced by $\mathrm{A} \beta$, and simultaneously to deal with the autoimmune activation that triggers acute brain inflammation. ${ }^{12-17}$ The AD vaccine tool kit was developed based on previous results obtained with the EB101 vaccine compound (Fig. ${ }^{15}$ ), formed by liposomes encapsulating phospholipids (phosphatidylcholine, phosphatidylglycerol, and cholesterol), into which a naturally-occurring immunostimulant adjuvant called sphingosine-1-phosphate (S1P) with neuroregenerator characteristics and the antigenic $A \beta 1-42$ protein is introduced. ${ }^{12-17}$

\section{Animals}

A well-studied mouse model of $\mathrm{A} \beta$ amyloidosis is the double$\mathrm{Tg}$ mice $\mathrm{B} 6 \mathrm{C} 3 \mathrm{~F} 1 / \mathrm{J}$ (APPswe/PS1dE9), expressing a chimeric mouse/human amyloid precursor protein (Mo/HuAPP695swe) and human presenilin 1 (PS1- $\Delta$ E9) mutants, both directed to central nervous system neurons, that exhibits $A \beta$ plaques in the hippocampus and cortex beginning at 6 months of age (Jackson Laboratory, Bar Harbor, ME, USA). All experimental procedures conformed to the guidelines established by the European Communities Council Directive (86/609/EEC), the EU Directive 2010/63/EU and the Spanish Royal Decree 1201/2005 for animal experimentation, and were approved by the Ethical Committee of the EuroEspes Biotechnology Research Centre (Permit number: $\mathrm{EE} / 2015-14$ ).

\section{Experimental design}

Two groups of experimental studies, preventive treatment (before amyloid deposition onset, starting at 7 weeks of age) and therapeutic treatment (after amyloid deposition onset at 35 weeks of age), were carried out as described in previous reports, ${ }^{12-17}$ similar to the long-term protocol reported by Schenk and colleagues. ${ }^{6}$ Mice of both sexes (balanced between treatment groups) were randomly assigned to each of the two experimental groups, and they were divided into two treatment groups per study. For the preventive treatment, Group A was formed by 10 mice $(7 \mathrm{Tg}$ and 3 wildtype mice) that were immunized with a cocktail of synthetic human A $342 / \mathrm{S} 1 \mathrm{P}$-containing liposomes (EB101), and Group B was formed by 10 mice ( $7 \mathrm{Tg}$ and 3 wild-type mice, control) inoculated with phosphate-buffered saline (PBS). For the therapeutic treatment, the same treatments were administrated in Groups A and B, formed by 10 mice in each group.

\section{Preparation of $A \beta$}

Two milligrams of human A $\beta 1-42$ (Tocris Bioscience, Tocris Cookson Ltd., Minneapolis, MN, USA), corresponding to the human form of the predominant $A \beta$ found in the brains of patients with $\mathrm{AD}$, were dissolved in $0.9 \mathrm{~mL}$ of autoclaved ultrapure water and brought up to $1 \mathrm{~mL}$ total volume by adding $0.1 \mathrm{~mL}$ of $10 \mathrm{x}$ PBS. This solution was vortexed, lyophilized and stored as a dry powder at $-20{ }^{\circ} \mathrm{C}$ until the preparation of liposomes.

\section{Preparation of liposomes}

Liposomes were prepared from 1,2-dioleoyl-sn-glycero-3-phosphocholine, 1-palmitoyl-2-oleoyl-sn-glycero-3-phosphatidylglycerol (sodium salt), and cholesterol (Northern Lipids Inc., Transferra Nanosciences Inc., Burnaby, BC, Canada), plus/minus D-erythro-S1P (at 0.3/0.3/0.39/0.01, molar ratio, respectively). A $100 \mathrm{mg}$ aliquot of each lipid was dissolved in $1 \mathrm{~mL}$ of chloroform and stored at $-20{ }^{\circ} \mathrm{C}$ until the preparation of the S1P liposomes, while $10 \mathrm{mg}$ was dissolved in $1.5 \mathrm{~mL}$ (2:1 chloroform/methanol) and stored at $-20{ }^{\circ} \mathrm{C}$ until use. Lipids, in the final proportion indicated above, were thoroughly mixed in the organic solvent and evaporated under nitrogen. The corresponding mixture, containing $100 \mathrm{mg}$ of total lipid and $0.6 \mathrm{mg}$ of S1P, was resuspended in autoclaved ultrapure water and thoroughly vortex-mixed until a milky solution was formed, the so-called "multilamellar vesicles". Small or single unilamellar vesicles were subsequently prepared by sonicating the multilamellar vesicles for $2 \mathrm{~m}$ at 30 -s intervals in an ice bath until the solution was clear, and then centrifuged at 2,500 g/15-m to eliminate debris. The SUV preparation was freeze-dried and resuspended in PBS-containing $\mathrm{A} \beta(10 \mathrm{mg})$, and thoroughly mixed. The freeze-dried mixture was resuspended in the corresponding amount of autoclaved ultrapure water, ready for immunization. A $100 \mu \mathrm{L}$ aliquot of EB101 was used for the im- 


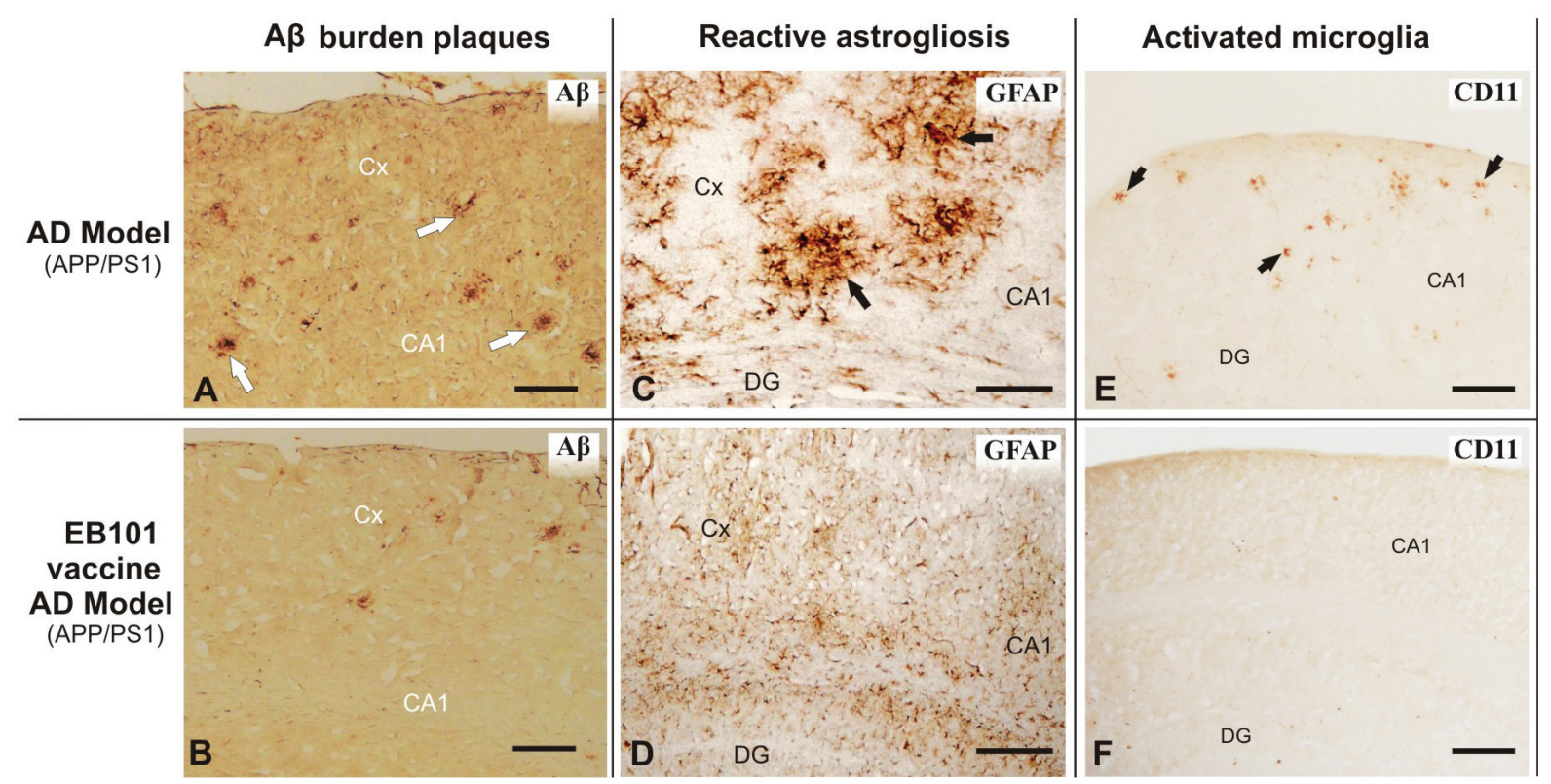

Fig. 2. Effect of EB101 vaccine on $A \beta$ deposits, astroglia and microglia in APP/PS1 mouse brain. Comparative photomicrographs of $A \beta$ immunoreactivity were taken in the cortical $(A-F)$ brain regions of transgenic $A D$ mice $(A, C, E)$ and after being treated with the $E B 101$ vaccine $(B, D, F)$ as immunotherapeutic treatment. Scale bar: $100 \mu \mathrm{m}$. A $\beta$, beta amyloid; AD, Alzheimer's disease.

munization step.

\section{Preparation of EB101 liposomal formulation}

We combined a biologically active lipid, S1P, with the antigenic A $\beta 1-42$ protein, an adjuvant previously used for liposomal immunization that has been successfully used for other vaccines, including influenza. This new liposomal vehicle acts as a matrix to solubilize and deliver A $\beta 1-42$ and $\mathrm{S} 1 \mathrm{P}$ as an adjuvant (Fig. 1).

\section{Immunization procedures}

Wild-type and $\mathrm{AD} \mathrm{Tg}$ mice were inoculated intraperitoneally with $100 \mu \mathrm{L}$ per injection of EB101 vaccine during 7 months (9 injections) for each treatment period. The immunization protocol was systematically used in previous similar studies and was followed strictly in accordance with the related published studies, ${ }^{21,22}$ with 3 injections every 2 weeks in the first month, and then 1 injection each following month. This immunization regime must be followed during the preventive and therapeutic periods.

\section{Immunohistochemistry analysis}

Immunohistochemical hallmarks were analyzed by using methods described in previous publications. ${ }^{12-17}$ In summary, parallel transverse sections $(12-14 \mu \mathrm{m})$ from the left half of the brain were sectioned by cryostat and pretreated with $\mathrm{H}_{2} \mathrm{O}_{2}$ in PBS at room temperature for $15 \mathrm{~m}$ to eliminate endogenous peroxidase. They were then rinsed twice in $0.05 \mathrm{M}$ Trizma-buffered saline (TBS) containing $0.1 \%$ Tween-20 at $\mathrm{pH} 7.4$ (TBS-T) for $10 \mathrm{~m}$ each, pretreated with an avidin/biotin blocking kit (Vector Labs, Burlingame, CA, USA) and then incubated overnight with the corresponding primary antibody $[A \beta 1-42$, mouse monoclonal 1:1,000, 05-831-I (Mil- lipore, Billerica, MA, USA); glial fibrillary acidic protein (GFAP), mouse monoclonal 1:100, G-3893 (Sigma, St. Louis, MO, USA); CD11/B-cells, rat polyclonal 1:500, MABF514 (Millipore)], as shown in Figure 2.

The sections were successively rinsed in TBS-T, incubated in goat IgG anti-fat (Millipore) or goat IgG anti-mouse (Sigma), depending on the primary antibody, for $1 \mathrm{~h}$, rinsed in TBS-T, and then incubated for $30 \mathrm{~m}$ using the $\mathrm{ABC}$ kit system (Vectastain; Vector Labs). The labeling was revealed by incubating sections with 3,3-diaminobenzidine (Sigma) as chromogen and hydrogen peroxide as oxidant. In several adjacent sections, negative controls (performed by omitting the primary, secondary or tertiary antibodies) showed no immunostaining.

Images were visualized using a microscope (BX50; Olympus, Tokyo, Japan) and digitized using a digital camera (DP-10; Olympus). The photographs were adjusted for brightness and contrast with Corel Photo-Paint (Corel 11, Ottawa, Canada) and figure images were composed using Corel Draw. Immunopositive hallmark quantitation was performed with pixcavator 4.0 software on the left half of the brain of each mouse. The left brain coronal sections were previously reconstituted from digital images acquired with a 10x-20x objective using the Photo-Merge tool in Corel Draw.

\section{Indications and usage}

EB101 is a lyophilized vaccine constituted by A $\beta 1-42$ and S1P emulsified in liposome complex, indicated for the prevention and treatment of $\mathrm{AD}$ pathological dysfunction in animal models (Fig $3)$.

\section{Dosage and administration}

EB101 is administered intraperitoneally, as indicated above. For most experimental protocols, the starting dose is $100 \mu \mathrm{g}$ in $100 \mu \mathrm{L}$ 


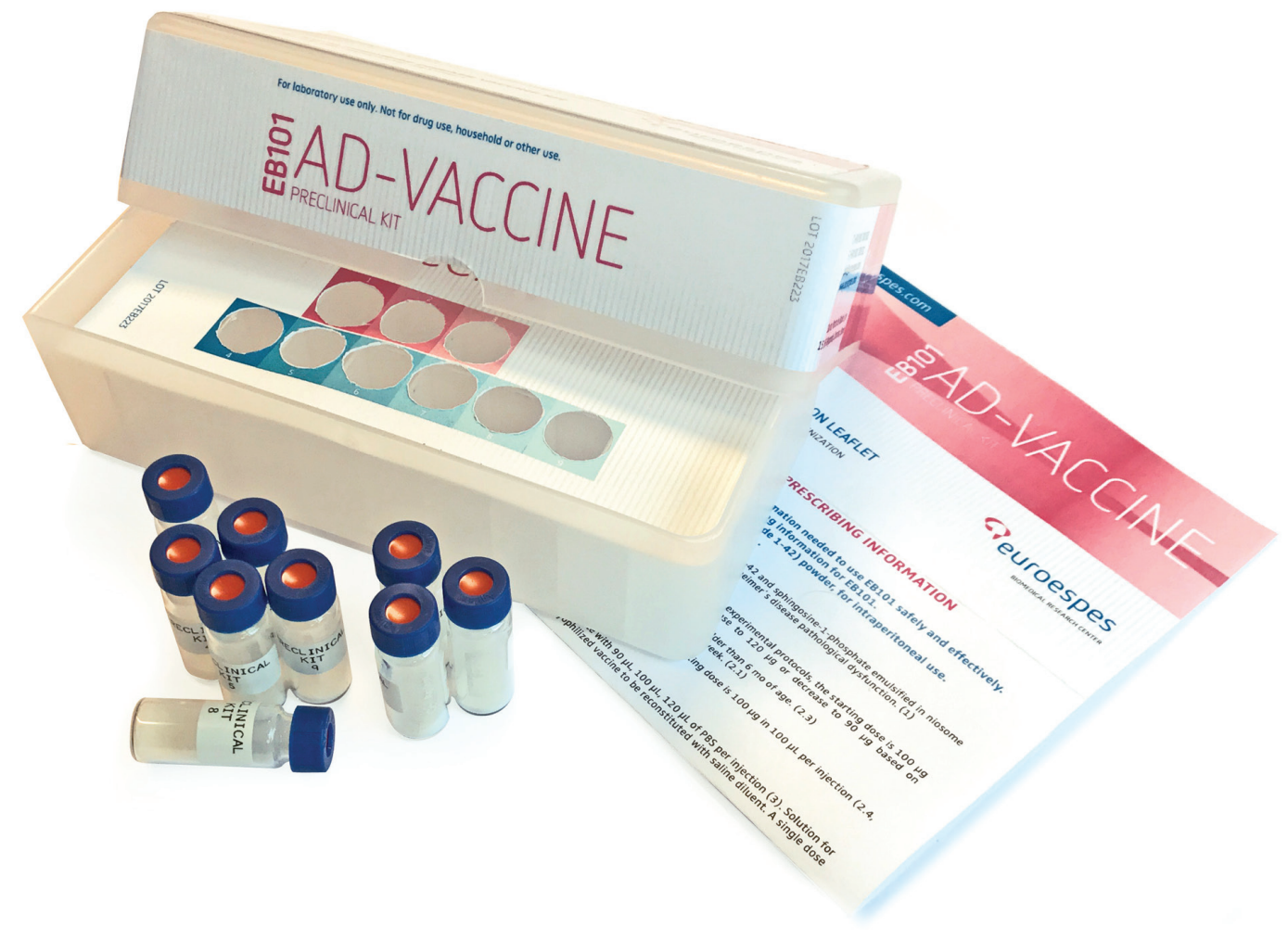

Fig. 3. Preclinical EB101 vaccine tool kit for animal models. Representative images of the EB101 vaccine tool kit for animal models, illustrating the different components of the kit via internal (left) and external (right) views.

of PBS per injection, up to once monthly. This is increased to 120 $\mu \mathrm{g}$ or decreased to $90 \mu \mathrm{g}$, based on efficacy/tolerability. A starting dose of $100 \mu \mathrm{L}$ of EB101 should be considered in mice older than 6 months of age. The maximum recommended dosing frequency is one injection per week. EB101 may be administered without adjuvants. In $\mathrm{AD}$ Tg mice older than 12 months, the recommended starting dose is $100 \mu \mathrm{g}$ in $100 \mu \mathrm{L}$ per injection.

\section{Dosage forms and strengths}

EB101 lyophilized powder $(100 \mu \mathrm{g})$ was reconstituted with $90 \mu \mathrm{L}$, $100 \mu \mathrm{L}$ or $120 \mu \mathrm{L}$ of PBS per injection. Solution for injection was supplied as a single-dose vial of lyophilized vaccine to be reconstituted. The single-dose after reconstitution was $100 \mathrm{~mL}$.

\section{Contraindications}

Contraindications included administration with complete Freund's adjuvant or other adjuvants, and severe allergic reaction (e.g., anaphylaxis) after a previous dose of any component of EB101.

\section{Results and discussion}

\section{Efficacy of $A D$ vaccine (EB101) in preventing and treating $A \beta$ burden}

We have recently designed, developed and tested a vaccine against the pathological effects of $\mathrm{AD}$ in mice, based on passive immunotherapy delivering A $\beta 1-42$ in a novel immunogen-adjuvant consisting of S1P-containing liposomes, to APP/PS1 Tg mice before and after detectable AD-like neuropathological hallmarks. Quantitative analysis of amyloid burden area and density, performed in the affected brain regions such as neocortex, entorhinal cortex and hippocampus, showed a remarkable decrease in $A \beta$ plaques, when EB101 vaccine was compared with other control treatments (Fig. 2). This finding demonstrates a key role as a regenerative-neuroprotective agent during the development and consolidation periods of AD neuropathology.

Therefore, results published by Carrera and colleagues demonstrated that EB101 vaccine significantly prevents and halts the development of $\mathrm{AD}$ neuropathological hallmarks in mice by inhibiting the generation of new $A \beta$ plaques, reducing dystrophic plaque neurites and minimizing neuroinflammation (Fig. 2), ${ }^{12-17}$ while reducing notably the neuronal degeneration and thus prolonging lifespan in Tg mice, paving the way for future clinical trials. Based on our previous studies, the preventive and therapeutic effects of the EB101 vaccine in APP/PS1 mice, designed to address the pitfalls of previous preclinical vaccines, preserve the immunogenicity targeted for the reduction of $A \beta$ burden, but avoid the massive activation of $\mathrm{T}$ cell-mediated immune response that potentially causes adverse inflammatory effects.

Efficacy of AD vaccine (EB101) in the immune response of APP/ PS1 mouse models

The immune response of $\mathrm{AD}$ vaccine in $\mathrm{Tg}$ mice was addressed by the quantification of astrocyte activation density in the hip- 
pocampal regions. Reactive astrocytes are known to be implicated in several pathological mechanisms, such as neuroinflammation, amyloidogenesis and neuronal cell death in AD. At the end of the preventive immunization, EB101 vaccine led to a significant reduction in the density of GFAP-reactive cell clusters in the hippocampal and neocortical regions, when compared with control treatments. Transverse brain sections of $\mathrm{AD} \mathrm{Tg}$ mice immunized with EB101 vaccine showed only a few scattered GFAP-reactive clusters, mainly at the outer cortical layers, contrasting with the numerous dystrophic reactive astrocytes observed in different cortical and hippocampal areas of mouse brains under control treatment (Fig. 2).

After therapeutic immunization with the EB101 vaccine, $A D$ $\mathrm{Tg}$ mouse brains were histopathologically similar to those of wildtype control mice, mostly devoid of astrogliosis and reactive clusters, except for a few scattered hallmarks in the intermediate cortical layers. No astrogliosis was observed in wild-type control mice during preventive or therapeutic treatment periods.

\section{Dual effect of the AD vaccine (EB101) in APP/PS1 mouse mod- els}

The data already published by Carrera and colleagues support the dual beneficial effect observed in all the experimental studies in which the EB101 vaccine was used as an immunotherapeutic agent in AD mouse models. ${ }^{12-17}$ The results indicate the potential benefits of EB101 immunization, emphasizing the effective A $\beta 1-42$ protein delivered in a novel immunogen-adjuvant composed of liposome-containing S1P, which induces a safe and effective antibody response, while preventing damaging neuroinflammation and ameliorating pathological brain degeneration in mice. The data collected during 5 years of preclinical research indicate that the chronic administration of EB101 vaccine to AD Tg mice led to a dual immunotherapeutic effect.

When AD Tg mice were immunized with EB101 vaccine at younger ages, before $A \beta$ plaques appeared, the preventive effect was observed since a notably reduced number of $A \beta$ plaques were developed at older ages. The dual effect was confirmed when EB101 vaccine induced a significant reduction of $A \beta$ accumulation in both cortical and hippocampal regions of AD Tg mice when $A \beta$ plaques had already developed. This therapeutic effect was also measured by different imaging and biochemical methods, leading to the experimental assumption that $\mathrm{AD}$ vaccine immunization protocol can be effective both before and after $\mathrm{A} \beta$ plaque development (Fig. 2). Therefore, immunization with EB101 has demonstrated a significant neuroprotective effect in the prevention and control of $\mathrm{AD}$-like neuropathology, by halting disease progression without developing behavioral deficits in the AD Tg mice tested. ${ }^{12-17}$

\section{EB101 AD vaccine immunization strategy in $\mathrm{Tg}$ mice}

The immunotherapeutic approach has become one of the most promising ways to prevent or treat $\mathrm{AD}$ and related neuropathological hallmarks, based on previous results of immunization strategies in Tg mice. ${ }^{23}$ Over the past decade, the goal of achieving an effective immunity rate against $\mathrm{A} \beta$ by means of a range of different antigenic designs and immunomodulatory strategies has been attempted, with varying success in $\mathrm{AD}$ mouse model studies. ${ }^{24-26}$ However, some of these studies have demonstrated that both passive and active immunization can lead to a significant reduction in amyloid deposition density and can even prevent the decline in cognitive performance in mice. ${ }^{27}$
At present, our EB101 vaccine is one of few vaccines against $\mathrm{AD}$ that has demonstrated effective results in $\mathrm{AD}$ Tg animal models genetically affected by the related pathological hallmarks. ${ }^{12-17}$ Moreover, recent advances in the development of liposome-based immunization techniques have demonstrated effective potential in the treatment of AD. ${ }^{28}$ Based on our previous studies, using active immunotherapy to reduce $A \beta$ plaque accumulation, ${ }^{12-17}$ here, we show a newly-developed vaccine tool kit to be applied to animal $\mathrm{AD}$ models in order to circumvent the previous vaccine failures in humans, due to an extensive T cell-mediated immune response. ${ }^{29}$

The present EB101 vaccine tool kit features a novel, proven formulation that generates autoimmunity against $A \beta 1-42$, and easily associates with the phospholipid-S1P-liposomes by the hydrationrehydration method. ${ }^{12-17}$ This methodology has been used previously in other immunization studies for an efficient liposomal-protein configuration, overcoming the immune pathological response of other types of adjuvants such as Freund's adjuvant,, ${ }^{6,26}$ Quil-A and the detergent polysorbate 80 to solubilize $A \beta$, which are believed to induce a proinflammatory Th 1 cell response. ${ }^{27,29,30}$

Experimental studies using $A \beta$ immunotherapy in $T g$ mouse models have revealed the induction of significantly high $\mathrm{A} \beta$ antibody levels in serum, depending on the type of vaccine adjuvant, mouse model, immunization methodology, and the level of response evoked by the adjuvant applied. ${ }^{31,32}$ These factors have been taken into consideration in the final formulation strategy for the development of a novel adjuvant-liposomal vaccine kit, in which the phospholipid-S1P-liposomes represent the pivotal structure of the lyophilized powder that provides an effective immunotherapeutic AD-related biomarker response, unlike previous vaccine formulations tested in AD-like mice. The remarkable effect of the EB101 immunization protocol, obtained in AD Tg mice after the establishment of $A \beta$ plaques in the hippocampus/neocortex and subsequent neuropathological changes, demonstrates that the EB101 vaccine tool kit presents a dramatic potential as a future therapeutic agent to effectively reverse the progression of pathological hallmarks. ${ }^{12-17}$

The experimental results obtained, both in the preventive and therapeutic treatments, demonstrated the dual effectivity of EB101 vaccine, not only in preventing the development of AD-like pathology but also in reducing its development once established. By using the EB101 vaccine tool kit in APP/PS1 Tg mice as preventive immunization, a significant reduction in density of the $A \beta$ plaque burden was observed in the affected regions, as well as reduced burden areas when compared with APP/PS1 mice treated with different vaccine components or PBS. Schenk and colleagues reported similar preventive immunization results in a mouse model of Alzheimer's disease (PDAPP) mice, ${ }^{6}$ although immunization efficiency and vaccine conformation have been improved in the present vaccine formulation.

The scientific community has accepted massive glial activation as a direct response to early development of $A \beta$ deposits in the $\mathrm{AD}$ brain, representing the main key to an efficient preventive immunization..$^{32-35}$ Accepting this statement, we have developed the EB101 vaccine under this hypothesis, reinforcing its effect on the prevention of the astrocyte activation process. Since the activation of astrocytes and microglia has been reported to induce the degradation of $A \beta,{ }^{36,37}$ the near-absence of $A \beta$-related astrocytosis in cortex and hippocampus of mice treated with EB101 vaccine confirmed the preventive effect against the development of ADlike pathology in this mouse model. ${ }^{12-17}$

Comparative $A \beta$ antibody titers and Th1/Th2 cytokine levels have been also analyzed in previous studies by enzyme-linked immunosorbent assay during mouse immunization. Data have showed that preventive treatment with EB101 resulted in a marked 
increase of specific $\operatorname{IgG} \mathrm{A} \beta 1-42$ antibody titers production, ${ }^{12-15}$ between 1:2,000 and 1:8,000, while the control group showed three and four times lower serum titer level $(p<0.01)$. The effect of EB101 vaccine on Th1 and Th2 cytokines was also studied. ${ }^{12-15}$ The EB101-treated group showed a tendency to Th1 secretion reduction, with respect to the control group, in both preventive and therapeutic treatments. The changes in Th2 protein levels followed the inverse trend, where the highest differences between the EB101-treated group and the control group were observed in the interleukin (IL)-4, IL-5 and IL-10 cytokine secretions $(p<0.05)$. The data indicates that EB101 vaccine resulted in an effective immunological response in $\mathrm{AD}$ mice models, including the induction of a Th2 cell response, which effectively prevented neuroinflammation.

These studies have provided further evidence to demonstrate that the EB101 vaccine tool kit (Fig. 3) is a reliable immunization agent in animal models used in $\mathrm{AD}$ research. However, the mechanism by which passive immunization blocks AD hallmarks is not yet well understood. ${ }^{38,39}$ One possibility is that the antibodies neutralize $A \beta$ in some restricted compartment or deplete the soluble form of $A \beta$ responsible for the pathological degeneration observed in the brain. ${ }^{40}$ In recent studies, soluble $A \beta$ forms have been proposed as the cause of synapse loss in APP Tg mice. ${ }^{41} \mathrm{~A}$ second possibility is the clearance effect of $A \beta$ deposits exerted by microglia, activated by immunizations. ${ }^{42}$ Based on our previous reports, ${ }^{12-17}$ the absence of adverse effects on behavior and brain functioning, and the neuroprotection in the affected area by the EB101 vaccine, we strongly recommend trying this $\mathrm{AD}$ vaccine tool kit for the treatment and prevention of $\mathrm{AD}$ in different animal models.

\section{Future research directions}

Currently, immunotherapy has become a reliable strategy and is starting to play a strong role not only in the preclinical research studies of degenerative molecular interaction but also in early diagnosis and treatment. ${ }^{43,44}$ However, it remains to be seen whether the antibody-based EB101 vaccine under development will have the high impact that is expected in other animal models, and probably in $\mathrm{AD}$ patients in a near future. The AD vaccine tool kit presentation (Fig. 3) described above, encourages optimism in which immunotherapy will surely constitute the best therapeutic treatment and prevention strategy against $\mathrm{AD}$ in the next decade.

An effective immunotherapy technique that can delay $\mathrm{AD}$ onset by 5 years would reduce the prevalence of the disease by half, while delaying its onset for 10 years would almost eradicate symptomatic AD. ${ }^{45}$ With the growing societal and governmental recognition of the importance of improving $\mathrm{AD}$ treatment, in the coming years this dramatic development process of passive vaccination for $\mathrm{AD}$ will hopefully bring some good news to the global health system.

\section{Conclusions}

We have shown above the beneficial dual effect of the EB101 AD vaccine tool kit derived from combining the liposomal/S1P adjuvant with an A $\beta 1-42$ antigen to induce an effective immunological response in a mouse model of $\mathrm{AD}$. Based on the numerous research studies performed, we can assume that the EB101 vaccine prevents or strongly reduces the main AD-like pathological hallmarks in the brain, while inhibiting astrocyte activation and the neuroinflamma- tory response that is expected to develop in $\mathrm{AD}$ Tg mice. However, further studies are needed in other AD animal models to effectively test the therapeutic approach of this preclinical vaccine formulation. We believe that understanding the biological mechanisms of this immunotherapeutic paradigm will speed up novel treatment regimens for $\mathrm{AD}$ patients.

\section{Conflict of interest}

The authors declare that they have no conflict of interests.

\section{Author contributions}

Study design (IC, RC), performance of experiments (IC), analysis and interpretation of data (IC, CV, RC), manuscript writing (IC, $\mathrm{RC})$, critical revision (CV, RC), statistical analysis (IC), critical funding (RC), administration (IC), technical or material support (IC, CV, RC).

\section{References}

1] Cacabelos R, Torrellas C, Teijido O, Carril JC. Pharmacogenetic considerations in the treatment ofAlzheimer's disease. Pharmacogenomics 2016;17(9):1041-1074. doi:10.2217/pgs-2016-0031.

[2] Cacabelos R, Torrellas C, Carrera I. Opportunities in pharmacogenomics for the treatmentof Alzheimer's disease. Future Neurology 2015;10(3):229-252. doi:10.2217/fnl.15.12.

[3] Cacabelos R, Martínez R, Fernández-Novoa L, Carril JC, Lombardi V, Carrera I, et al. Genomics of dementia: APOE- and CYP2D6-related pharmacogenetics. Int J Alzheimers Dis 2012;2012:518901. doi:10.1155/2012/518901.

[4] Blennow K, de Leon M, Zetterberg H. Alzheimer's disease. Lancet 2006;368(9533):387-403. doi:10.1016/S0140-6736(06)69113-7.

[5] Puzzo D, Lee L, Palmeri A, Calabrese G, Arancio O. Behavioral assays with mouse models of Alzheimer'sdisease: practical considerations and guidelines. Biochem Pharmacol 2014;88(4):450-467. doi:10.1016/j.bcp.2014.01.011.

[6] Schenk D, Barbour R, Dunn W, Gordon G, Grajeda H, Guido T, et al. Immunization with amyloid-beta attenuates Alzheimer-disease-likepathology in the PDAPP mouse. Nature 1999;400(6740):173-177. doi:10.1038/22124.

[7] Davtyan H, Bacon A, Petrushina I, Zagorski K, Cribbs DH, Ghochikyan $A$, et al. Immunogenicity of DNA- and recombinant proteinbasedAlzheimer Disease epitope vaccines. Hum Vaccin Immunother 2014;10(5):1248-1255. doi:10.4161/hv.27882.

[8] Carrera I, Novoa L, Teijido O, Sampedro C, Seoane S, Lakshmana $\mathrm{M}$, et al. Comparative characterization profile of transgenicmouse models of Alzheimeŕs disease. J Genomic Med Pharmacogenomics 2017;2(1):331-337.

[9] Jindal H, Bhatt B, Sk S, Singh Malik J. Alzheimer disease immunotherapeutics: then and now. Hum Vaccin Immunother 2014;10(9):27412743. doi:10.4161/21645515.2014.970959.

[10] Lannfelt L, Relkin NR, Siemers ER. Amyloid-ß-directed immunotherapy for Alzheimer'sdisease. J Intern Med 2014;275(3):284-295. doi:10.1111/joim.12168.

[11] Delrieu J, Ousset PJ, Caillaud C, Vellas B. 'Clinical trials in Alzheimer's disease':immunotherapy approaches. J Neurochem 2012;120(Suppl 1):186-193. doi:10.1111/j.1471-4159.2011.07458.x.

[12] Carrera I, Etcheverría I, Fernández-Novoa L, Lombardi V, Cacabelos R, Vigo C. Vaccine development to treat Alzheimer's diseaseneuropathology in APP/PS1 transgenic mice. Int J Alzheimers Dis 2012;2012:376138. doi:10.1155/2012/376138.

[13] Carrera I, Cacabelos R. Novel immunotherapeutic procedures for prevention ofAlzheimeŕs disease. Drug Des 2013;2:107. 
doi:10.4172/2169-0138.1000107.

[14] Carrera I, Etcheverría I, Li Y, Fernández-Novoa L, Lombardi V, Vigo $\mathrm{C}$, et al. Immunocytochemical characterization of Alzheimer diseasehallmarks in APP/PS1 transgenic mice treated with a new anti-amyloid- $\beta$ vaccine. Biomed Res Int 2013;2013:709145. doi:10.1155/2013/709145.

[15] Carrera I, Etcheverría I, Fernández-Novoa L, Lombardi VR, Lakshmana $\mathrm{MK}$, Cacabelos $\mathrm{R}$, et al. A comparative evaluation of a novel vaccine in APP/PS1mouse models of Alzheimer's disease. Biomed Res Int 2015;2015:807146. doi:10.1155/2015/807146.

[16] Carrera I, Fernandez-Novoa L, Aliev G, Vigo C, Cacabelos R. Validating immunotherapy in Alzheimer's disease:The EB101 vaccine. Curr Pharm Des 2016;22(7):849-858.

[17] Carrera I, Fernández-Novoa L, Vigo C, Cacabelos R. Sphingosine1-phosphate (S1P)-Containing Niosomes asVehicle to Deliver Amyloid- $\beta$ in a Novel Alzheimeŕs DiseaseVaccine. J Genomic Med Pharmacogenomics 2016;1:225-232.

[18] Masliah E, Hansen L, Adame A, Crews L, Bard F, Lee C, et al. Abeta vaccination effects on plaque pathology in theabsence of encephalitis in Alzheimer disease. Neurology 2005;64(1):129-131. doi:10.1212/01. WNL.0000148590.39911.DF.

[19] Nicoll J, Wilkinson D, Holmes C, Steart P, Markham H, Weller RO. Neuropathology of human Alzheimer disease after immunizationwith amyloid-beta peptide: a case report. Nat Med 2003;9(4):448452. doi:10.1038/nm840.

[20] Ferrer I, Boada Rovira M, Sánchez Guerra ML, Rey MJ, Costa-Jussá F. Neuropathology and pathogenesis of encephalitis followingamyloid-beta immunization in Alzheimer's disease. Brain Pathol 2004;14(1):11-20. doi:10.1111/j.1750-3639.2004.tb00493.x.

[21] Zhou J, Fonseca MI, Kayed R, Hernandez I, Webster SD, Yazan O, et al. Novel $A ß$ peptide immunogens modulate plaque pathologyand inflammation in a murine model of Alzheimer's disease. J Neuroinflammation 2015;2:28. doi:10.1186/1742-2094-2-28.

[22] Wisniewski T, Boutajangout A. Immunotherapeutic approaches for Alzheimer'sdisease in transgenic mouse models. Brain Struct Funct 2010;214(2-3):201-218. doi:10.1007/s00429-009-0236-2.

[23] Rafii MS. Update on Alzheimer's disease therapeutics. Rev Recent Clin Trials 2013;8(2):110-118. doi:10.2174/1574887111308999004 5 .

[24] Davtyan H, Petrushina I, Ghochikyan A. Immunotherapy for Alzheimer's disease: DNA- andprotein-based epitope vaccines. Methods Mol Biol 2014;1143:259-281. doi:10.1007/978-1-4939-0410-5_16.

[25] Wisniewski T, Goñi F. Immunotherapy for Alzheimer's disease. Biochem Pharmacol 2014;88(4):499-507. doi:10.1016/j. bcp.2013.12.020.

[26] Wilcock DM, Gharkholonarehe N, Van Nostrand WE, Davis J, Vitek MP, Colton CA. Amyloid reduction by amyloid- $\beta$ vaccination alsoreduces mouse tau pathology and protects from neuron loss in two mousemodels of Alzheimer's disease. J Neurosci 2009;29(25):79577965. doi:10.1523/JNEUROSCI.1339-09.2009.

[27] Panza F, Frisardi V, Solfrizzi V, Imbimbo BP, Logroscino G, Santamato $A$, et al. Immunotherapy for Alzheimer's disease: from anti$\beta$-amyloidto tau-based immunization strategies. Immunotherapy 2012;4(2):213-238. doi:10.2217/imt.11.170.

[28] Agrawal M, Ajazuddin, Tripathi DK, Saraf S, Saraf S, Antimisiaris SG, et al. A. Recent advancements in liposomes targeting strategiesto cross blood-brain barrier (BBB) for the treatment of Alzheimer'sdisease. J Control Release 2017;260:61-77. doi:10.1016/j.jconrel.2017.05.019.

[29] Ghochikyan A, Mkrtichyan M, Petrushina I, Movsesyan N, Karapetyan A, Cribbs DH, et al. Prototype Alzheimer's disease epitope vaccineinduced strong Th2-type anti-Abeta antibody response with
Alum toQuil A adjuvant switch. Vaccine 2006;24(13):2275-2282. doi:10.1016/j.vaccine.2005.11.039.

[30] Gilman S, Koller M, Black RS, Jenkins L, Griffith SG, Fox NC, et al. Clinical effects of Abeta immunization (AN1792) inpatients with AD in an interrupted trial. Neurology 2005;64(9):1553-1562. doi:10.1212/01. WNL.0000159740.16984.3C.

[31] Jankowsky JL, Fadale DJ, Anderson J, Xu GM, Gonzales V, Jenkins NA, et al. Mutant presenilins specifically elevate the levelsof the 42 residue beta-amyloid peptide in vivo: evidence for augmentationof a 42-specific gamma secretase. Hum Mol Genet 2003;13(2):159-170. doi:10.1093/hmg/ddh019.

[32] Trinchese F, Liu S, Battaglia F, Walter S, Mathews PM, Arancio O. Progressive age-related development of Alzheimer-likepathology in APP/ PS1 mice. Ann Neurol 2004;55:801-814. doi:10.1002/ana.20101.

[33] Davtyan H, Ghochikyan A, Petrushina I, Hovakimyan A, Davtyan A, Poghosyan $A$, et al. Immunogenicity, efficacy and mechanism of action ofepitope vaccine for Alzheimer's disease: prelude to clinicaltrial. J Neurosci 2013;33(11):4923-4934. doi:10.1523/JNEURO$\mathrm{SCl} .4672-12.2013$.

[34] Cacabelos R, Meyyazhagan A, Carril JC, Cacabelos P, Teijido Ó. Pharmacogenetics of vascular risk factors in Alzheimer'sdisease. J Pers Med 2018;8(1):pii:E3. doi:10.3390/jpm8010003.

[35] Cacabelos R, Martínez-Bouza R. Genomics and pharmacogenomics of dementia. CNS Neurosci Ther 2011;17(5):566-576. doi:10.1111/ j.1755-5949.2010.00189.x.

[36] Morales I, Guzmán-Martínez L, Cerda-Troncoso C, Farías GA, Maccioni RB. Neuroinflammation in the pathogenesis of Alzheimer'sdisease. A rational framework for the search of novel therapeuticapproaches. Front Cell Neurosci 2014;8:112. doi:10.3389/fncel.2014.00112.

[37] Birch AM, Katsouri L, Sastre M. Modulation of inflammation in transgenic models ofAlzheimer's disease. J Neuroinflammation 2014;11:25. doi:10.1186/1742-2094-11-25.

[38] Barrera-Ocampo A, Lopera F. Amyloid-beta immunotherapy: the hope for Alzheimerdisease?. Colomb Med (Cali) 2016;47(4):203212.

[39] Delrieu J, Ousset PJ, Voisin T, Vellas B. Amyloid beta peptide immunotherapy in Alzheimer disease. Rev Neurol 2014;170(12):739-748. doi:10.1016/j.neurol.2014.10.003.

[40] Subramanian S, Savanur G, Madhavadas S. Passive immunization targeting the $\mathrm{N}$-terminal regionof phosphorylated tau (residues 68-71) improves spatial memory inokadaic acid induced tauopathy model rats. Biochem Biophys Res Commun 2017;483(1):585-589. doi:10.1016/j.bbrc.2016.12.101.

[41] Mucke L, Masliah E, Yu GQ, Mallory M, Rockenstein EM, Tatsuno G, et al. High level neuronal expression of abeta 1-42in wild-type human amyloid precursor protein precursor transgenicmice: Synaptotoxicity without plaque formation. J Neurosci 2000;20(11):4050-4058.

[42] Morgan D, Diamond DM, Gottschall PE, Ugen KE, Dickey C, Hardy J, et al. A beta peptide vaccination prevents memory loss inan animal model of Alzheimer's disease. Nature 2000;408(6815):982-985. doi:10.1038/35050116

[43] Cacabelos R, Torrellas C. Epigenetics of aging and Alzheimer's disease:Implications for pharmacogenomics and drug response. Int J Mol Sci 2015;16(12):30483-30543. doi:10.3390/ijms161226236.

[44] Cacabelos R, Torrellas C, Carrera I, Cacabelos P, Corzo L, FernándezNovoa L, et al. Novel therapeutic strategies for dementia. CNS Neurol Disord Drug Targets 2016;15(2):141-241. doi:10.2174/18715273156 66160202121548.

[45] Brookmeyer R, Johnson E, Ziegler-Graham K, Arrighi HM. Forecasting the global burden of Alzheimer'sdisease. Alzheimers Dement 2007;3(3):186-191. doi:10.1016/j.jalz.2007.04.381. 